\title{
Long-Wavelength MHD Instability in the Prefront of Collisionless Shocks with Accelerated Particles
}

\author{
A. M. Bykov ${ }^{1 *}$, S. M. Osipov ${ }^{2}$, and I. N. Toptygin ${ }^{3}$ \\ ${ }^{1}$ Ioffe Physicotechnical Institute, Russian Academy of Sciences, \\ ul. Politekhnicheskaya 26, St. Petersburg, 194021 Russia \\ ${ }^{2}$ Academic Physicotechnological University, Russian Academy of Sciences, St. Petersburg, Russia \\ ${ }^{3}$ St. Petersburg State Polytechnical University, \\ ul. Politekhnicheskaya 29, St. Petersburg, 195251 Russia \\ Received October 16, 2008
}

\begin{abstract}
Collisionless shocks in turbulent space plasmas accelerate particles by the Fermi mechanism to ultrarelativistic energies. The interaction of accelerated particles with the plasma inflow produces extended supersonic MHD flows of multicomponent plasma. We investigate the instabilities of a flow of threecomponent turbulent plasma with relativistic particles against long-wavelength perturbations with scales larger than the accelerated particle transport mean free path and the initial turbulence scales. The presence of turbulence allows us to formulate the system of single-fluid equations, the equation of motion for the medium as a whole, and the induction equation for the magnetic field with turbulent magnetic and kinematic viscosities. The current of accelerated particles enters into the induction equation with an effective magnetic diffusion coefficient. We have calculated the local growth rates of the perturbations related to the nonresonant long-wavelength instability of the current of accelerated particles for MHD perturbations in the WKB approximation. The amplification of long-wavelength magnetic field perturbations in the flow upstream of the shock front can affect significantly the maximum energies of the particles accelerated by a collisionless shock and can lead to the observed peculiarities of the synchrotron X-ray radiation in supernova remnants.
\end{abstract}

PACS numbers: $94.20 . \mathrm{Wc} ; 98.38 . \mathrm{Mz} ; 98.70 . \mathrm{Sa}$

DOI: $10.1134 / \mathrm{S} 1063773709080052$

Key words: MHD instability, accelerated particles

\section{INTRODUCTION}

The processes of rapid energy release in plasmas are often accompanied by supersonic flows and shock formation. Shocks play a special role in shaping the nonthermal radiation spectra and generating the magnetic fields in various objects. A long series including gamma-ray bursts (Meszaros 2006), supernova remnants (Reynolds 2008), clusters of galaxies, and intergalactic medium (Bykov et al. 2008a; Ryu et al. 2008) provides examples.

The Chandra observations of nonthermal structures in supernova remnants dominated by continuum X-ray emission probably of a synchrotron origin are interpreted as observational evidence for the effects of fast particle acceleration to energies above $10^{14} \mathrm{eV}$ with the simultaneous superadiabatic amplification of the local magnetic field approximately by two orders of magnitude by a collisionless shock

\footnotetext{
${ }^{*}$ E-mail: byk@astro.ioffe.ru
}

(see the review by Reynolds (2008) and references therein). The proper interpretation of observational data requires modeling and understanding the nonthermal processes in collisionless shocks.

Some fraction of the kinetic energy of supersonic and super-Alfvénic flows is converted into the thermal energy of the plasma through collective plasma processes. As a result, a collisionless shock front is formed. The collective relaxation is accompanied by the excitation of a large number of degrees of freedom with a strong coupling between the plasma modes and particles (Sagdeev 1964; Boyd and Sanderson 2003). The strong perturbations of the electromagnetic field of short-lived plasma modes dissipate inside the viscous subshock region. The width of the viscous subshock $\Delta_{\text {sh }}$ at which part of the directed flow velocity is thermalized through collective processes, the plasma is compressed, and, accordingly, the transverse magnetic field is amplified reaches several hundred inertial ion lengths $l_{\mathrm{i}}=c / \omega_{\mathrm{pi}} \approx 2.3 \times$ 
$10^{7} n^{-0.5} \mathrm{~cm}$ (here, the plasma density is measured in $\mathrm{cm}^{-3}$ ), depending on the magnetic field inclination.

Upstream of the dissipative viscous subshock region, the conversion of plasma flow energy into particle energy continues via the scattering of a group of suprathermal particles by long-lived Alfvén MHD oscillations frozen into the preshock plasma flow. The possibility of an efficient acceleration of energetic nonthermal particles is an important distinctive feature of the shocks in extended cosmic objects.

A small fraction of particles is accelerated by the Fermi mechanism to energies that exceed the kinetic energies of the particles of the supersonic plasma flow in a collisionless shock in a turbulent medium by many orders of magnitude on macroscopic length scales $l_{\mathrm{f}} \sim c / u_{\mathrm{sh}} \times \Lambda(p)$. Here, $u_{\mathrm{sh}}$ is the plasma flow velocity relative to the shock front and $\Lambda(p)$ is the transport mean free path of an energetic particle with momentum $p$ in a turbulent magnetic field. Energetic nonthermal particles penetrate to a depth of the order of $l_{\mathrm{f}}$ and modify the preshock plasma flow by producing a prefront. The size of the prefront $\sim l_{\mathrm{f}}$ exceeds the width of the viscous subshock $\Delta_{\text {sh }}$ by many orders of magnitude. Modeling supersonic and super-Alfvénic flows including the relativistic particle acceleration effects with both Particle-in-Cell (PIC) and hybrid codes requires a self-consistent allowance for the nonlinear processes in three dimensions and on greatly differing scales, $\Delta_{\text {sh }}$ and $l_{\mathrm{f}}$. The first successful PIC models of collisionless shocks with Fermi particle acceleration were obtained only for relativistic shocks by Spitkovsky (2008). Modeling nonrelativistic shocks requires a much higher computer power.

The shocks in the shells of young supernova remnants have typical velocities of $\sim 0.1-0.01 \quad c$ and accelerate particles to energies of $\sim 100 \mathrm{TeV}$ (Reynolds 2008). Modeling such shocks with PIC (and hybrid) codes is unachievable on modern computers (for a discussion, see Vladimirov et al. 2008). An MHD description of turbulent supersonic flows and averaged nonthermal particle distribution functions are commonly used. Strongly nonlinear models of such shocks can be constructed by the Monte Carlo method in which the relationship of the particle transport mean free path to the local macroscopic flow parameters and MHD turbulence properties are postulated a priori (Vladimirov et al. 2006). Parameterizing the transport mean free path in a Monte Carlo model requires a separate analysis of the MHD turbulence properties on the microscopic level.

Nonequilibrium accelerated particle distributions can be unstable and can amplify the fluctuations of various scales in the prefront. The resonant excitation of oscillations by anisotropic distributions of relativistic particles underlies most of the models for cosmicray propagation (Skilling 1975; Berezinskii et al. 1990; Blandford and Eichler 1987; Kulsrud 2005). In this case, anisotropic flows of relativistic particles amplify the modes in resonance with the particle gyrofrequencies. The plasma instabilities related to the presence of an accelerated particle pressure gradient were investigated by Dorfi and Drury (1985), Berezhko (1986), Chalov (1988), and Malkov and Drury (2001) in terms of a model approach to describe the accelerated particle pressure.

Nonresonant plasma instabilities related to the presence of an external current of energetic particles are also possible. Bell (2004) pointed out that MHD fluctuations with wavelengths smaller than or of the order of the gyroradii $r_{g}\left(p_{1}\right)$ of the lowest-energy accelerated particles, i.e., under the condition $k r_{g}\left(p_{1}\right)>1$, could be amplified. Below, we call this limit the short-wavelength current instability. Bell (2004) estimated the saturation level of the magnetic field amplitude in an unstable shortwavelength mode, $\delta B_{\mathrm{sat}} / 8 \pi \sim u_{\mathrm{sh}} / c \times P_{\mathrm{CR}}$. The nonthermal particle pressure $P_{\mathrm{CR}}$ in the case of an efficient particle acceleration by a collisionless shock can account for a sizeable fraction (more than 10\%) of the kinetic energy density of the plasma flow on the collisionless shock front. Thus, according to the estimate by Bell (2004), $\delta B_{\text {sat }}$ in a strong collisionless shock can exceed appreciably the unperturbed magnetic field strength in the collisionless shock prefront and can affect both the width of the viscous subshock in the collisionless shock and the maximum energies of the accelerated particles. A dramatic growth of short-wavelength longitudinal MHD modes was also obtained in the numerical nonlinear MHD models investigated by Zirakashvili and Ptuskin (2008). The first results of the simulations of a short-wavelength instability using a threedimensional plasma code (Niemiec et al. 2008) are also indicative of the presence of instability but they give a slower growth of the longitudinal MHD mode than that predicted by Bell's quasi-linear analysis. The longitudinal short-wavelength fluctuations in the model are saturated at the level of an unperturbed longitudinal field $\delta B_{\mathrm{sat}} \sim B_{0}$ with the formation of a strongly discontinuous filamentary magnetic field structure (Niemiec et al. 2008). A dramatic growth of oblique perturbations was found in the model. A further study of the nonlinear evolution of the shortwavelength instability is needed for a fuller understanding of the mechanisms and saturation levels of the nonresonant perturbations. Pelletier et al. (2006) and Marcowith et al. (2006) discussed the formation of MHD turbulence spectra in the presence of a Bell instability with anisotropic cascades and pointed to a 
possible modification of the Fermi acceleration related to efficient field generation.

The current instability can also be accompanied by the nonresonant generation of long-wavelength fluctuations when the suppression mechanisms of the transverse plasma conductivity are present (Bykov and Toptygin 2005, 2007; Reville et al. 2007). In this case, perturbations with a wavelength exceeding the mean free path of energetic particles $\Lambda(p)$ grow. The long-wavelength instabilities attributable to the presence of a current of energetic particles external with respect to the background (quasi-thermal) plasma differ from the instabilities of a background plasma with an anisotropic Weibel (1959) velocity distribution. The current of energetic particles induces currents and fields in the background plasma, which admit a linear instability when the external current shielding suppression mechanisms are present, in particular, through an anomalous increase in magnetic diffusion by turbulence. Turbulent magnetic diffusion of the plasma reduces the shielding of the external current of accelerated particles producing the instability in the background plasma. The magnetic diffusion can be increased by small-scale electromagnetic fluctuations (see, e.g., Moffat 1980; Vainshtein et al. 1980; Bykov and Toptygin 1993).

In the papers devoted to the current instability, the Alfvénic perturbations propagating along the normal to the front of a longitudinal collisionless shock related to the anisotropy of energetic particles and maintained through their free energy were investigated.

Here, we investigate the possibilities for realizing a nonresonant long-wavelength instability of MHD fluctuations with wavelengths exceeding both the gyroradius of a particle with momentum $p$ in a mean effective magnetic field and the transport mean free path $\Lambda(p)$ of energetic particles with momentum $p$ (i.e., $k \Lambda(p)<1$ ) in supersonic and super-Alfvénic turbulent plasma flows. Long-wavelength fluctuations with $k r_{g}(p) \ll 1$ can be amplified by the instability of the current of energetic particles if the magnetic diffusion is increased significantly by fluctuations with scales $k r_{g}\left(p_{1}\right)>1$.

The amplified long-wavelength fluctuations will resonantly scatter the highest-energy $\left(\sim p_{\max }\right)$ particles and, hence, their simulations are fundamentally important in estimating the maximum energies of the particles accelerated by the Fermi mechanism. Moreover, turbulent magnetic fields increase dramatically the efficiency and lead to temporal variations in the synchrotron radiation of ultrarelativistic electrons accelerated in supernova remnants (see Bykov et al. 2008b). Rapidly varying synchrotron radiation in the X-ray energy band is observed from some supernova remnants (Uchiyama et al. 2007).

\section{EQUATIONS OF MOTION \\ FOR A TURBULENT PLASMA \\ WITH NONTHERMAL PARTICLES}

To investigate the stability, we will derive a system of equations that reduces the dynamics of the long-wavelength perturbations of a collisionless turbulent plasma with energetic particles to a singlefluid model. To this end, we will average the equations of plasma motion over fluctuations with scales smaller than the mean free path of the accelerated energetic particles. In particular, the Bell (2004) instability can be a source of small-scale fluctuations. We will average the equations for the background plasma and nonthermal particles over the MHD fluctuations and calculate the turbulent viscosity and magnetic diffusion coefficients by the methods of mean-field electrodynamics widely used in the dynamo theory (for a detailed review, see Brandenburg and Subramanian 2005).

\section{Averaging the Equations of Motion and Magnetic Induction over Small-Scale Fluctuations}

Let us derive the magnetic induction equation averaged over fluctuations with scales smaller than the gyroradius of the accelerated particles. We will perform the averaging by the method suggested by Blackman and Field (2002). After the separation of the fields in scales $\mathbf{B}=\overline{\mathbf{B}}+\mathbf{b}$ and $\mathbf{V}=\overline{\mathbf{V}}+\mathbf{v}$, where $\overline{\mathbf{B}}$ and $\overline{\mathbf{V}}$ are the large-scale magnetic field and velocity of the medium, we will write the equation for the large-scale quantities:

$$
\frac{\partial \overline{\mathbf{B}}}{\partial t}=c \nabla \times \overline{\mathbf{E}}+\nabla \times(\overline{\mathbf{V}} \times \overline{\mathbf{B}})+\nu_{m} \triangle \overline{\mathbf{B}} .
$$

Here, $\nu_{m}$ is the magnetic viscosity, $c \overline{\mathbf{E}}=\langle\mathbf{v} \times \mathbf{b}\rangle$ is the turbulent electromotive force, $\mathbf{v}$ and $\mathbf{b}$ are the small-scale magnetic field and velocity of the medium, the brackets \langle\rangle denote averaging over the small-scale fluctuations. The electromotive force $\overline{\mathbf{E}}$ can be found from the dynamic equation

$$
c \frac{\partial \overline{\mathbf{E}}}{\partial t}=\left\langle\frac{\partial \mathbf{v}}{\partial \mathbf{t}} \times \mathbf{b}\right\rangle+\left\langle\mathbf{v} \times \frac{\partial \mathbf{b}}{\partial \mathbf{t}}\right\rangle .
$$

The equation for the small-scale fluctuations of the background plasma velocity in our case differs from that used by Blackman and Field (2002) by the presence of a current of accelerated particles in the problem. This current at the averaging stage in question is external and affects the magnetic field via the Maxwell equations including the Ohm law:

$$
\begin{gathered}
\operatorname{curl} \mathbf{B}=\frac{4 \pi}{c}\left(\mathbf{j}+\mathbf{J}^{\mathrm{cr}}\right), \\
\mathbf{J}^{\mathrm{cr}}=\overline{\mathbf{j}}^{\mathrm{cr}}+\mathbf{j}^{\mathrm{cr}}, \quad \mathbf{E}=-\frac{1}{c}(\mathbf{V} \times \mathbf{B}) .
\end{gathered}
$$


The equation of motion for the background plasma is

$$
\begin{gathered}
\rho\left(\frac{\partial \mathbf{V}}{\partial t}+(\mathbf{V} \nabla) \mathbf{V}\right) \\
=-\nabla P+\frac{1}{c}(\mathbf{j} \times \mathbf{B})+e\left(n_{i}-n_{e}\right) \mathbf{E}+\nu \triangle \mathbf{V} ;
\end{gathered}
$$

we assume the medium as a whole to be quasineutral, $n_{i}+n_{\mathrm{cr}}=n_{e}$.

To simplify our calculations, following Blackman and Field (2002), we assume the small-scale modes to be incompressible. To obtain $\partial \overline{\mathbf{E}} / \partial t$ in Eq. (2), we need an equation for the small-scale velocity, which we derive from (4) using (3),

$$
\begin{aligned}
\frac{\partial v_{q}}{\partial t}= & \hat{P}_{q i}\left\{-\frac{1}{c \rho}\left(\overline{\mathbf{j}}^{\mathrm{cr}} \times \mathbf{b}\right)_{i}+\frac{e n_{\mathrm{cr}}}{c \rho}(\mathbf{v} \times \overline{\mathbf{B}})_{i}\right. \\
& +\frac{1}{4 \pi \rho}(\overline{\mathbf{B}} \nabla) b_{i}+\frac{1}{4 \pi \rho}(\mathbf{b} \nabla) B_{i} \\
+ & \frac{e n_{\mathrm{cr}}}{c \rho}(\overline{\mathbf{V}} \times \mathbf{b})_{i}-(\overline{\mathbf{V}} \nabla) v_{i}-(\mathbf{v} \nabla) \overline{V_{i}} \\
& -(\mathbf{v} \nabla) v_{i}+\left\langle(\mathbf{v} \nabla) v_{i}\right\rangle+\frac{1}{4 \pi \rho}(\mathbf{b} \nabla) b_{i} \\
& \left.-\frac{1}{4 \pi \rho}\left\langle(\mathbf{b} \nabla) b_{i}\right\rangle\right\}+\nu \Delta v_{q}+f_{q},
\end{aligned}
$$

where $\hat{P}_{q i} \equiv\left(\delta_{q i}-\nabla^{-2} \nabla_{q} \nabla_{i}\right)$ is the projection operator that provides incompressibility in the NavierStokes equation, $f_{q}$ is the part of the force in Eq. (5) uncorrelated with $\mathbf{b}$, and $\overline{\mathbf{j}}_{\text {cr }}$ is the large-scale current of cosmic rays. We neglect the fluctuations in the current of cosmic rays on scales much smaller than the gyroradius of the accelerated particles (see also Bell 2004), but below we will take into account the large-scale variations in this current. Here, $n_{\mathrm{cr}}$ is the number density of the accelerated particles (these are assumed to be protons), $e$ is the elementary charge, $\rho$ is the background plasma density, and $\nu$ is the viscosity. The equation for the small-scale magnetic field is

$$
\begin{aligned}
& \frac{\partial \mathbf{b}}{\partial t}=(\overline{\mathbf{B}} \nabla) \mathbf{v}-(\mathbf{v} \nabla) \overline{\mathbf{B}}+\nabla \times(\overline{\mathbf{V}} \times \mathbf{b}) \\
& +\nabla \times(\mathbf{v} \times \mathbf{b})-\nabla \times\langle\mathbf{v} \times \mathbf{b}\rangle+\nu_{m} \triangle \mathbf{b}
\end{aligned}
$$

Next, we will consider the small-scale turbulence produced by the Bell (2004) instability. We will assume that the turbulence consists of growing modes with the wave vector directed along the local largescale field $\overline{\mathbf{B}}$ and this direction will be chosen as the $z$ axis. According to Bell (2004), the dispersion relation for this instability can be derived from the following equations for the transverse (to the wave vector) components of the small-scale field:

$$
\begin{aligned}
& \left(\omega^{2}-\bar{v}_{a}^{2} k_{z}^{2}\right) b_{x}+i \frac{\left(\bar{j}_{z}^{\mathrm{cr}}-e n_{\mathrm{cr}} \bar{V}_{z}\right) \bar{B}_{z}}{c \rho} k_{z} b_{y}=0, \\
& \left(\omega^{2}-\bar{v}_{a}^{2} k_{z}^{2}\right) b_{y} \\
& -i \frac{\left(\bar{j}_{z}^{\mathrm{cr}}-e n_{\mathrm{cr}} \bar{V}_{z}\right) \bar{B}_{z}}{c \rho} k_{z} b_{x}=0,
\end{aligned}
$$

where $\bar{v}_{a}=B_{z} / \sqrt{4 \pi \rho}$. Thus, from (7) we obtain the dispersion relation

$$
\omega^{2}=\bar{v}_{a}^{2}\left(k_{z}^{2}-k_{1} k_{z}\right), k_{1}=\frac{4 \pi}{c} \frac{\left(\bar{j}_{z}^{\mathrm{cr}}-e n_{\mathrm{cr}} \bar{V}_{z}\right)}{\bar{B}_{z}} .
$$

For a growing mode at $\left(\bar{j}_{z}^{\mathrm{cr}}-e n_{\mathrm{cr}} \bar{V}_{z}\right)>0$ and $\bar{B}_{z}>0$,

$$
\omega=i \bar{v}_{a} \sqrt{k_{1}\left|k_{z}\right|-k_{z}^{2}} .
$$

At the stage of linear growth, we have the following relation between the amplitudes of the Fourier harmonics of the small-scale field $\mathbf{b}_{\mathbf{k}}$ and velocity $\mathbf{v}_{\mathbf{k}}$ from the induction equation:

$$
i \omega \mathbf{b}_{\mathbf{k}}=-i \bar{B}_{z} k_{z} \mathbf{v}_{\mathbf{k}} .
$$

Using the fact that $k_{1} \gg\left|k_{z}\right|$ for the region in $k$ with the greatest mode amplification and Eqs. (9) and (10), we obtain

$$
\left|\mathbf{v}_{\mathbf{k}}\right|^{2} \approx \frac{1}{4 \pi \rho} \frac{k_{1}}{\left|k_{z}\right|}\left|\mathbf{b}_{\mathbf{k}}\right|^{2} .
$$

Substituting (9) into (7) yields the polarization of the growing mode

$$
b_{x}=i \frac{k_{z}}{\left|k_{z}\right|} b_{y} .
$$

Using (9), (11), and (12), we will obtain the Fourier transforms of the correlators of the smallscale quantities

$$
\begin{gathered}
\left\langle b_{\alpha}^{*}(\mathbf{k}) b_{\beta}(\mathbf{k})\right\rangle=\frac{\left\langle\mathbf{b}^{2}\left(k_{z}\right)\right\rangle}{2} \\
\times \delta\left(k_{x}\right) \delta\left(k_{y}\right) \times\left(\begin{array}{ccc}
1 & -i \frac{k_{z}}{\left|k_{z}\right|} & 0 \\
i \frac{k_{z}}{\left|k_{z}\right|} & 1 & 0 \\
0 & 0 & 0
\end{array}\right), \\
\left\langle v_{\alpha}^{*}(\mathbf{k}) v_{\beta}(\mathbf{k})\right\rangle=\frac{1}{4 \pi \rho} \frac{k_{1}}{k_{z}} \frac{\left\langle\mathbf{b}^{2}\left(k_{z}\right)\right\rangle}{2} \\
\times \delta\left(k_{x}\right) \delta\left(k_{y}\right)\left(\begin{array}{ccc}
1 & -i \frac{k_{z}}{\left|k_{z}\right|} & 0 \\
i \frac{k_{z}}{\left|k_{z}\right|} & 1 & 0 \\
0 & 0 & 0
\end{array}\right),
\end{gathered}
$$

ASTRONOMYLETTERS Vol. 35 No. 82009 


$$
\begin{aligned}
&\left\langle v_{\alpha}^{*}(\mathbf{k}) b_{\beta}(\mathbf{k})\right\rangle= \frac{1}{\sqrt{4 \pi \rho}} \sqrt{\frac{k_{1}}{k_{z}}} \frac{\left\langle\mathbf{b}^{2}\left(k_{z}\right)\right\rangle}{2} \\
& \times \delta\left(k_{x}\right) \delta\left(k_{y}\right)\left(\begin{array}{ccc}
i \frac{k_{z}}{\left|k_{z}\right|} & 1 & 0 \\
-1 & i \frac{k_{z}}{\left|k_{z}\right|} & 0 \\
0 & 0 & 0
\end{array}\right), \\
&\left\langle b_{\alpha}^{*}(\mathbf{k}) v_{\beta}(\mathbf{k})\right\rangle=-\left\langle v_{\alpha}^{*}(\mathbf{k}) b_{\beta}(\mathbf{k})\right\rangle .
\end{aligned}
$$

Substituting (5) and (6) into (2) and performing averaging using (13)-(15) yields the equation for the electromotive force

$$
\begin{gathered}
\frac{\partial \overline{\mathbf{E}}}{\partial t}=-\langle\mathbf{v} \cdot \nabla \times \mathbf{v}\rangle \bar{B}_{z} \mathbf{e}_{z} \\
+\frac{1}{\rho c}\left\langle\mathbf{b}^{2}\right\rangle\left(\bar{j}_{z}^{\mathrm{cr}}-e n_{\mathrm{cr}} \bar{V}_{z}\right) \mathbf{e}_{z} \\
+\frac{\left\langle v^{2}\right\rangle}{2}\left(\frac{\partial \bar{B}_{z}}{\partial y} \mathbf{e}_{x}-\frac{\partial \bar{B}_{z}}{\partial x} \mathbf{e}_{y}\right)+\ldots
\end{gathered}
$$

where the ellipsis denotes the triple correlators and $\mathbf{e}_{x}, \mathbf{e}_{y}, \mathbf{e}_{z}$ are unit vectors along the corresponding axes. In deriving Eq. (16), we discarded the terms containing correlators (13) and (15), except the term containing the current of accelerated particles, because these correlators are small compared to correlator (14) at $k_{1} \gg\left|k_{z}\right|$. Below, we will also omit the terms containing the derivatives of the largescale quantities with respect to the $x$ and $y$ coordinates, because these derivatives are small for the lowamplitude perturbations of the large-scale quantities under consideration.

Using (14), we obtain the relation

$$
-\langle\mathbf{v} \cdot \nabla \times \mathbf{v}\rangle=\frac{1}{\rho c}\left\langle\mathbf{b}^{2}\right\rangle \frac{\left(\bar{j}_{z}^{\mathrm{cr}}-e n_{\mathrm{cr}} \bar{V}_{z}\right)}{\bar{B}_{z}} .
$$

Following the method and using (1), (16), and (17), we find the induction equation for the largescale magnetic field (here, the large-scale current of accelerated particles and magnetic field were assumed to be coaligned)

$$
\begin{aligned}
\frac{\partial \overline{\mathbf{B}}}{\partial t}= & \nabla \times(\overline{\mathbf{V}} \times \overline{\mathbf{B}})+\frac{2}{\rho c} \tau_{\mathrm{cor}}\left\langle\mathbf{b}^{2}\right\rangle \nabla \\
& \times\left(\overline{\mathbf{j}}_{\mathrm{cr}}-e n_{\mathrm{cr}} \overline{\mathbf{V}}\right)+\nu_{m} \triangle \overline{\mathbf{B}},
\end{aligned}
$$

where $\tau_{\text {cor }}$ is the effective correlation time introduced into mean-field electrodynamics by the method of Blackman and Field (2002). The coefficient $\frac{\left\langle\mathbf{b}^{2}\right\rangle}{4 \pi \rho} \tau_{\text {cor }}=\nu_{\mathrm{s}}$ characterizes the turbulent diffusion of the mean transverse magnetic field and determines the shielding of the external current in mean-field electrodynamics.
Note that the presence of a current of accelerated particles in Eq. (5), which leads to the instability of small-scale Bell modes with a relation between the velocity and field amplitudes different from that for Alfvén modes, is important in deriving Eq. (18). Averaging over the small-scale modes gives the second (current) term and the nonzero first term on the right-hand side of Eq. (16) responsible for the longwavelength instability that we study below.

Averaging the terms $\langle(\mathbf{v} \nabla) \mathbf{v}\rangle$ and $\langle(\mathbf{b} \nabla) \mathbf{b}\rangle$ in the Navier-Stokes equation for the large-scale quantities similarly to the averaging $\langle\mathbf{v} \times \mathbf{b}\rangle$ yields the following equation for the large-scale plasma velocity:

$$
\begin{gathered}
\frac{\partial \overline{\mathbf{V}}}{\partial t}+(\overline{\mathbf{V}} \nabla) \overline{\mathbf{V}}=-\frac{1}{\rho} \nabla P-\frac{1}{c \rho}\left(\overline{\mathbf{j}}^{\mathrm{cr}} \times \overline{\mathbf{B}}\right) \\
+\frac{e n_{\mathrm{cr}}}{c \rho}(\overline{\mathbf{V}} \times \overline{\mathbf{B}})+\frac{1}{4 \pi \rho}((\nabla \times \overline{\mathbf{B}}) \times \overline{\mathbf{B}}) \\
-\tau_{\mathrm{cor}} \frac{1}{c \rho}\left(\frac{\partial \bar{j}_{z}^{\mathrm{cr}}}{\partial x} \mathbf{e}_{x}+\frac{\partial \bar{j}_{z}^{\mathrm{cr}}}{\partial y} \mathbf{e}_{y}\right) \int_{0}^{\infty} d k_{z} \frac{1}{\sqrt{4 \pi \rho}} \\
\times \sqrt{\frac{k_{1}}{k_{z}}}\left\langle\mathbf{b}^{2}\left(k_{z}\right)\right\rangle+\tau_{\mathrm{cor}}\langle\mathbf{v} \cdot \nabla \times \mathbf{v}\rangle \\
\times\left(\frac{\partial \bar{V}_{z}}{\partial y} \mathbf{e}_{x}-\frac{\partial \bar{V}_{z}}{\partial x} \mathbf{e}_{y}\right)+\tau_{\mathrm{cor}} \frac{\left\langle v^{2}\right\rangle}{2}\left(\frac{\partial^{2} \overline{\mathbf{V}}}{\partial x^{2}}+\frac{\partial^{2} \overline{\mathbf{V}}}{\partial y^{2}}\right) \\
+\nu \triangle \overline{\mathbf{V}}-\frac{1}{c \rho}\left\langle\left(\mathbf{j}^{\mathrm{cr}}-e n_{\mathrm{cr}} \mathbf{V}\right) \times \mathbf{b}\right\rangle \ldots
\end{gathered}
$$

Below, in Eq. (23), we neglect the terms containing the derivatives of the large-scale quantities with respect to the transverse $x$ and $y$ coordinates, which are small when the growth rates of longitudinal lowamplitude large-scale perturbations are calculated.

After averaging Eq. (19) over fluctuations with scales larger than the gyroradius of the accelerated particles and their mean free path (these two parameters are close in the Bohm limit), the last term of this equation has the meaning of an elastic collision integral via the small-scale fluctuations with the accelerated particles. Let us consider the hydrodynamic equation for the accelerated particles

$$
\frac{\partial \mathcal{P}^{(r)}}{\partial t}+\nabla \cdot \widehat{\Pi}^{(r)}=\frac{1}{c}\left(\mathbf{J}^{\mathrm{cr}} \times \mathbf{B}\right)+e n_{\mathrm{cr}} \mathbf{E},
$$

where the momentum flux density is expressed in terms of the accelerated particle distribution function $F_{r}$ as

$$
\Pi_{\alpha \beta}^{(r)}=\int v_{\alpha} p_{\beta} F_{r} d^{3} p=\rho_{r} u_{\alpha}^{(r)} u_{\beta}^{(r)}+P_{\mathrm{cr}} \delta_{\alpha \beta} .
$$

Given (3), Eq. (20) after averaging over the small scales reads

$$
\left\langle\frac{\partial \mathcal{P}^{(r)}}{\partial t}+\nabla \cdot \widehat{\Pi}^{(r)}\right\rangle=\frac{1}{c}\left(\overline{\mathbf{j}}^{\mathrm{cr}} \times \overline{\mathbf{B}}\right)-e n_{\mathrm{cr}}(\overline{\mathbf{V}} \times \overline{\mathbf{B}})
$$




$$
+\frac{1}{c}\left\langle\left(\mathbf{j}^{\mathbf{c r}}-e n_{\mathrm{cr}} \mathbf{v}\right) \times \mathbf{b}\right\rangle .
$$

Adding (19) and (22) divided by $\rho$ term by term, we will obtain the equation of motion for the system as a whole. This equation is valid on scales larger than the mean free path of the accelerated particles for the scattering by magnetic fluctuations, where the hydrodynamic approximation for the relativistic particle momentum flux can be properly used:

$$
\begin{gathered}
\frac{\partial \overline{\mathbf{V}}}{\partial t}+(\overline{\mathbf{V}} \nabla) \overline{\mathbf{V}}=-\frac{1}{\rho} \nabla\left(P+P_{\mathrm{cr}}\right) \\
+\frac{1}{4 \pi \rho}((\nabla \times \overline{\mathbf{B}}) \times \overline{\mathbf{B}})+\nu \triangle \overline{\mathbf{V}}
\end{gathered}
$$

In contrast to the small-scale magnetic field fluctuations, the long-wavelength fluctuations induce significant perturbations of the current of accelerated particles $\delta \mathbf{j}^{\mathrm{cr}}$. Below, we will seek the growth rates of low-amplitude large-scale perturbations using the relations between the variations $\delta \mathbf{j}^{\mathrm{cr}}, \delta \mathbf{b}$, and $\mathbf{u}$ in the current of accelerated particles, the magnetic field, and the velocity of the medium propagating along a constant magnetic field $B_{0}$ directed along the normal to the shock front. We use a coordinate system in which the direction of the $z$ axis coincides with that of the constant magnetic field $B_{0}$.

\section{The Current of Accelerated Particles Produced by a Long-Wavelength MHD Perturbation}

Below, we will seek the growth rate of lowamplitude modes. To close the system of equations requires finding the perturbation $\delta \mathbf{j}^{(r)}$ of the current of accelerated particles produced by a weak MHD perturbation $\delta \mathbf{b}$ of the magnetic field.

In the small-scale domain, the perturbation of the current of accelerated particles can be derived from the collisionless kinetic equation for the accelerated particle momentum distribution function $f(p) \sim$ $p^{-4-\varepsilon}$ with $p_{0} \leq p \leq p_{\max }$ :

$$
\mathbf{j}_{\perp}^{\mathrm{cr}}=\sigma \frac{j_{\|}}{B_{\|}} \mathbf{B}_{\perp},
$$

where

$$
\begin{gathered}
\sigma=(1+\varepsilon)\left(r_{g} k_{\|}\right)^{1+\varepsilon} \int_{0}^{\frac{1}{r_{g} k_{\|}}} \lambda^{\varepsilon} \\
\times\left\{\frac{3}{4} \lambda\left(1-\lambda^{2}\right)\left[\ln \left(\frac{1+\lambda}{1-\lambda}\right)+i \pi\right]+\frac{3}{2} \lambda^{2}\right\} d \lambda,
\end{gathered}
$$

where $r_{g}=\frac{c p_{0}}{e B_{\|}}$is the gyroradius of the accelerated particles with the lowest momentum. In the simplest case of the spectrum of particles accelerated by a shock with a small distortion of the flow profile by the accelerated particle pressure, $\varepsilon \rightarrow 0$, we have $\sigma \approx\left(r_{g} k_{\|}\right)^{-2} \ll 1$ for small-scale fluctuations with $k_{\|} r_{g} \gg 1$. This allows the fluctuations in the current of relativistic particles to be neglected in the regime of small-scale instability (see Bell 2004; Pelletier et al. 2006). However, for long-wavelength perturbations with $k_{\|} \Lambda(p) \leq 1$, the variations in the current of relativistic particles and the magnetic field are strongly correlated (Bykov and Toptygin 2007) and this correlation essentially determines the pattern of the long-wavelength instability.

We will calculate the current of accelerated particles on large scales using the kinetic equation that describes the particle scattering by magnetic fluctuations,

$$
\begin{gathered}
\frac{\partial F_{r}}{\partial t}+\mathbf{v} \cdot \frac{\partial F_{r}}{\partial \mathbf{r}}+e \mathbf{E} \cdot \frac{\partial F_{r}}{\partial \mathbf{p}} \\
-\frac{e c}{\mathcal{E}}\left(\mathbf{B}_{0}+\delta \mathbf{b}\right) \cdot \mathcal{O} F_{r}=I\left[F_{r}\right],
\end{gathered}
$$

where $\mathcal{O}$ is the momentum rotation operator; $\mathbf{E}, \delta \mathbf{b}$ is the external electromagnetic field of the MHD wave; $F_{r}(\mathbf{r}, \mathbf{p}, t)$ is the accelerated particle distribution function; and $I\left[F_{r}\right]$ is the collision integral (see Toptygin 1983).

We will linearize the kinetic equation (26) by assuming the external field to be weak and by separating out the small part $\delta f$ attributable to this field from the distribution function $F_{r}=f_{0}+\delta f$ :

$$
\begin{aligned}
& \frac{\partial \delta f}{\partial t}+\mathbf{v} \cdot \frac{\partial \delta f}{\partial \mathbf{r}}-\frac{e c}{\mathcal{E}} \mathbf{B}_{0} \cdot \mathcal{O} \delta f \\
= & -e \mathbf{E} \cdot \frac{\partial f_{0}}{\partial \mathbf{p}}+\frac{e c}{\mathcal{E}} \delta \mathbf{b} \cdot \mathcal{O} f_{0}+I[\delta f] .
\end{aligned}
$$

The unperturbed distribution function is

$$
f_{0}(p, \theta)=\frac{1}{4 \pi}\left[N(p)+\frac{3 \mathbf{u}_{0 \|} \cdot \mathbf{v}}{v^{2}} N(p)\right],
$$

where $\mathbf{u}_{0||}$ is the velocity with which the medium flows onto the shock front and $N(p)$ is the isotropic part of the distribution function. In what follows, we will consider power-law relativistic particle momentum distributions:

$$
\begin{gathered}
N(p)=(\alpha-3) N_{0} \frac{p_{0}^{\alpha-3}}{p^{\alpha}} e^{u_{\|} z / \kappa} \Theta\left(p-p_{0}\right), \\
\alpha \geq 4, \quad p_{m} \geq p \geq p_{0} \approx m_{i} c .
\end{gathered}
$$

The last term in (27) accounts for the relaxation of the distribution function $\delta f$ through the interaction of accelerated particles with background particles and stochastic fields. We will represent it in the relaxation-frequency approximation: $I[\delta f] \rightarrow-\nu \delta f$. 
Here, we assume a low level of large-scale turbulence, i.e., $\nu \ll \frac{e B_{0}}{p}$; in this case, we consider perturbation wavelengths larger than the minimum gyroradius of the accelerated particles and neglect contributions of the order of $\frac{k_{\|} c}{\omega_{B_{p}}}$, where $\omega_{B_{p}}=\frac{e B_{0}}{p_{0}}$ is the gyrofrequency of the accelerated particles with the minimum magnitude of the momentum $p_{0}=m_{p} c$. Solving this problem in the same way as in the review by Bykov and Toptygin (2007), we find a linear (with respect to the magnetic field $\delta \mathbf{b}$ ) relation,

$$
\delta \mathbf{j}^{\mathrm{cr}}=g^{\prime} \delta \mathbf{b},
$$

with the kinetic coefficient

$$
g^{\prime}=\frac{e u_{0 \| \mid} n_{\mathrm{cr}}}{B_{0}} .
$$

Equation (30) with (31) is similar to (24) for $k_{\|} r_{g} \ll$ 1 , i.e., at $\sigma \approx 1$. The kinetic coefficient $g^{\prime}$ is a pseudoscalar, since the current is written via the magnetic induction pseudo-vector. On the right-hand side of (31), the projection of the polar vector onto the direction of the regular magnetic field $\mathbf{B}_{0}$, i.e., $u_{0 \|}$ is a pseudo-scalar.

\section{THE LOCAL GROWTH RATE OF MHD OSCILLATIONS}

Let us derive the expression for the local growth rate of Alfvén modes from Eqs. (18), (23), and the continuity equation

$$
\frac{\partial \rho}{\partial t}+\nabla(\rho \overline{\mathbf{V}})=0
$$

by taking into account Eq. (30) for the perturbation of the current of accelerated particles. We consider the simplest type of perturbation in the form of an Alfvén mode with the wave vector $k_{\|} \mathbf{e}_{0}$ along the normal to the longitudinal collisionless shock front.

Substituting the low-amplitude perturbations of the large-scale quantities in the form $\delta \mathbf{b}=\overline{\mathbf{B}}-\mathbf{B}_{0}$, $\delta \mathbf{v}=\overline{\mathbf{V}}$, and $\delta \mathbf{j}=\overline{\mathbf{j}}^{\text {cr }}-\mathbf{j} \|$ into Eqs. (18), (23), and (32) using (30) and eliminating $\delta \mathbf{v}$, we obtain the following relations for the transverse components of the large-scale magnetic field perturbation:

$$
\begin{gathered}
{\left[\omega^{2}-v_{\mathrm{a}}^{2} k_{\|]}^{2}\right] \delta b_{x}-i(8 \pi / c) \nu_{\mathrm{s}} g^{\prime} k_{\|} \omega \delta b_{y}=0,} \\
{\left[\omega^{2}-v_{\mathrm{a}}^{2} k_{\|]}^{2}\right] \delta b_{y}+i(8 \pi / c) \nu_{\mathrm{s}} g^{\prime} k_{\|} \omega \delta b_{x}=0,}
\end{gathered}
$$

where $v_{\mathrm{a}}=B_{0} / \sqrt{4 \pi \rho}$ is the Alfvén velocity.

Setting the determinant of system (33) equal to zero, we find the dispersion relation

$$
\omega^{2}-v_{\mathrm{a}}^{2} k_{\|}^{2} \pm i \frac{8 \pi}{c} \nu_{\mathrm{s}} g^{\prime} k_{\|} \omega=0 .
$$

The imaginary part of the frequency $\gamma=\Im \omega$ is defined by the relation

$$
\gamma(k)=\mp \nu_{\mathrm{s}} \frac{4 \pi k_{\|}}{c} g^{\prime} .
$$

Substituting (31) into (35) yields the expression for the growth rate

$$
\gamma(k)=\nu_{\mathrm{s}} k_{0} k_{\|}, \quad k_{0}=\frac{4 \pi}{c} \frac{e u_{0 \|} n_{\mathrm{cr}}}{B_{0}} .
$$

The growing mode has the polarization $\delta b_{y 1}=$ $i \delta b_{x 1}$ and it is opposite to the polarization of the small-scale modes over which the averaging was performed. Averaging over the large-scale turbulence will make a contribution to the helicity density $\langle\mathbf{u} \cdot \nabla \times \mathbf{u}\rangle>0$. Thus, when averaging over the developed large-scale turbulence, the term $-\langle\mathbf{u}$. $\nabla \times \mathbf{u}\rangle \nabla \times \overline{\mathbf{B}}$ will appear in the magnetic field induction (18) that will compensate for the contribution from the current term. Hence, following Taylor (1986), we can estimate the saturation level of the large-scale field related to the compensation effect,

$$
\left\langle\delta \mathbf{b}^{2}\right\rangle \bar{\tau}_{\text {cor }} k_{\|} \sim\left\langle\mathbf{b}^{2}\right\rangle \tau_{\text {cor }} k_{0},
$$

where $\bar{\tau}_{\text {cor }}$ is the correlation time on large scales;

$$
k_{0}=\frac{M_{\mathrm{A}}}{2} \frac{\omega_{\mathrm{pi}}}{c} \frac{n_{\mathrm{cr}}}{n_{\mathrm{i}}},
$$

where $\omega_{\mathrm{pi}}$ is the plasma ion frequency, $M_{\mathrm{A}}$ is the Alfvén Mach number of the shock, and $n_{\mathrm{i}}$ is the background plasma ion number density. Note that nonlinear effects can change the saturation level of the harmonic amplitudes.

We will estimate the growth rate of longwavelength perturbations by noting that the quantity $\tau_{\text {cor }} \sqrt{\frac{\left\langle\mathbf{b}^{2}\right\rangle}{4 \pi \rho}}=\frac{\xi}{k_{0}}$ is the effective mixing length of the small-scale turbulence (where $\xi$ is a dimensionless parameter):

$$
\gamma(k)=\sqrt{\frac{\left\langle\mathbf{b}^{2}\right\rangle}{4 \pi \rho}} \xi k_{\|} .
$$

The growth rate of the small-scale Bell instability responsible for the formation of magnetic field fluctuations $\left\langle\mathbf{b}^{2}\right\rangle$ determining the shielding of the current of accelerated particles has a maximum at $k_{s m}=\frac{k_{0}}{2}$. If the original spectrum of small-scale fluctuations declined with increasing $k_{s m}$, then $\xi>1$ are also possible. Since the small-scale modes grow only in a region smaller than the gyroradius of the accelerated particles with the minimum momentum and at a wave number $k_{s m}$ smaller than its critical value $\left(k_{0}\right)$, we have $k_{0}^{-1}<k_{s m}^{-1}<r_{g}$. Note that $k_{0}$ depends on 
the current of accelerated particles and the constant magnetic field (36) and, thus, the formation condition for the small-scale instability needed for the realization of turbulent transport coefficients is

$$
\frac{4 \pi}{c} \frac{e u_{0 \| \mid} n_{\mathrm{cr}}}{B_{0}}>r_{g}^{-1} \text {. }
$$

It is also important to keep in mind that the polarization pattern of the small-scale modes defined by Eq. (12) plays an important role in calculating $\nu_{s}$.

The current of accelerated particles is localized in the shock prefront region with size $l_{\mathrm{f}}$ and, hence, the instability region is also finite. The convective drift time of the fluctuations on the shock front can be estimated from the formula

$$
\tau_{d} \approx \frac{c \Lambda}{3 u_{0 \|}^{2}} .
$$

We find from (39) and (41) that a noticeable growth of unstable fluctuations with a wave number $k_{\|}$requires

$$
\gamma(k) \tau_{d}=\frac{\xi}{3} \frac{c \sqrt{\frac{\left\langle\mathbf{b}^{2}\right\rangle}{4 \pi \rho}}}{u_{0 \|}^{2}} k_{\|} \Lambda>1 .
$$

It follows from estimate (39) that the shocks in the shells of young supernova remnants can amplify long-wavelength magnetic field fluctuations with scales larger than the mean free paths of the accelerated relativistic particles by a nonresonant mechanism. The growth rate of the long-wavelength fluctuations is proportional to the turbulent magnetic viscosity. Strong turbulent fluctuations with scales smaller than the gyroradii of the accelerated relativistic particles, the possibility of whose amplification was investigated by Bell (2004) (see also Pelletier et al. 2005; Zirakashvili and Ptuskin 2008; Niemiec et al. 2008), play a significant role in increasing considerably the turbulent magnetic viscosity compared to the effects attributable to the finite (even anomalous) conductivity. The growth rates of the nonresonant current mechanism described by Eqs. (39) and (41) allow long-wavelength magnetic field fluctuations with scales larger than $10^{15} \mathrm{~cm}$ in supernova remnants with shock velocities $u_{0 \|} \sim$ $0.01 \mathrm{~s}$ to be amplified in a time of the order of a year.

Long-wavelength magnetic field fluctuations ensure a much more efficient scattering of relativistic particles than fluctuations with scales $k r_{g}(p) \gg$ 1. The transport mean free path of the relativistic particles scattered by small-scale inhomogeneities with $k r_{g}(p) \gg 1$ increases rapidly with an increase in momentum $\Lambda(p) \propto p^{2}$ (Toptygin 1983) incompatible with the Bohm diffusion traditionally used in the models of particle acceleration by shocks. The scattering of particles by long-wavelength magnetic field fluctuations usually provides the regimes of longitudinal diffusion $\Lambda(p) \propto p^{a}$ with indices $a \leq 1$, which, in particular, is important in modeling the spectra of the highest-energy nuclei accelerated in supernova remnants.

\section{CONCLUSIONS}

Below, we summarize our main results.

(1) We obtained the system of equations that reduced the dynamics of the long-wavelength perturbations of a collisionless turbulent three-component plasma (electrons, background and energetic ions) to a single-fluid model. By the long-wavelength perturbations we mean those with scales exceeding the mean free path of relativistic particles for the scattering by magnetic fluctuations. The relaxation of the system that makes the single-fluid description possible proceeds through momentum and energy exchange between the plasma components via electromagnetic field fluctuations.

(2) The presence of developed small-scale turbulence was shown to lead to rapid turbulent magnetic diffusion that suppresses significantly the shielding of the large-scale current of relativistic particles and, as a result, to the growth of a long-wavelength instability determined by this current. The mean helicity density of the amplified long-wavelength modes is opposite in sign to the helicity density produced by small-scale modes.

(3) The instability under consideration is nonresonant and all energetic particles, not only those resonant with the wave being excited, are involved in the amplification of the oscillations. This circumstance is particularly important in the case of strong turbulence, which distorts the helical trajectories of the accelerated particles and removes them from a narrow resonance.

(4) We restricted ourselves to the calculation of linear instability growth rates and to the application of the effect considered to collisionless shocks that efficiently accelerate particles. The magnetic field of the growing modes is perpendicular to the unperturbed magnetic field upstream of the shock front. This can be a factor that limits the nonlinear field growth. Constructing a nonlinear theory is beyond the scope of this paper.

(5) The long-wavelength turbulence amplification mechanism considered is universal and, in addition to the plasma flows related to collisionless shocks, it can be efficient in another very important class of flows, accretion plasma flows onto gravitating centers, in the presence of a source of accelerated particles. 


\section{ACKNOWLEDGMENTS}

We are grateful to the referees for constructive remarks. This work was supported in part by the Russian Foundation for Basic Research (project no. 09-02-00507a).

\section{REFERENCES}

1. A. R. Bell, Mon. Not. R. Astron. Soc. 353, 550 (2004).

2. E. G. Berezhko, Pis'ma Astron. Zh. 12, 842 (1986) [Sov. Astron. Lett. 12, 352 (1986)].

3. V. S. Berezinskii, S. V. Bulanov, V. L. Ginzburg, et al., Astrophysics of Cosmic Rays (Nauka, Moscow, 1990) [in Russian].

4. E. G. Blackman and G. B. Field, Phys. Rev. Lett. 89, 265007 (2002).

5. R. D. Blandford and D. Eichler, Phys. Rep. 154, 1 (1987).

6. T. J. M. Boyd and J. J. Sanderson, The Physics of Plasma (Cambridge Univ., Cambridge, 2003).

7. A. Brandenburg and K. Subramanian, Phys. Rep. 417, 1 (2005).

8. A. M. Bykov, K. Dolag, and F. Durret, Space Sci. Rev. 134, 119 (2008a).

9. A. M. Bykov and I. N. Toptygin, Usp. Fiz. Nauk 163, 19 (1993) [Phys. Usp. 36, 1020 (1993)].

10. A. M. Bykov and I. N. Toptygin, Pis'ma Astron. Zh. 31, 839 (2005) [Astron. Lett. 31, 748 (2005)].

11. A. M. Bykov and I. N. Toptygin, Usp. Fiz. Nauk 177, 149 (2007) [Phys. Usp. 50, 141 (2007)].

12. A. M. Bykov, Yu. A. Uvarov, and D. C. Ellison, Astrophys. J. 689, L133 (2008b).

13. E. A. Dorfi and L. O'C. Drury, Proc. 19 Intern. Cosmic Ray Conf. 3, 121 (1985).

14. R. M. Kulsrud, Plasma Physics for Astrophysics (Princeton Univ., Princeton, 2005).

15. M. A. Malkov and L. O'C. Drury, Rep. Progr. Phys. 64, $429(2001)$.

16. A. Marcowith, M. Lemoine, and G. Pelletier, Astron. Astrophys. 453, 192 (2006).
17. P. Meszaros, Rep. Progr. Phys. 69, 2259 (2006).

18. G. Moffat, Magnetic Field Excitation in a Conducting Medium (Mir, Moscow, 1980), p. 150 [in Russian].

19. J. Niemiec, M. Pohl, T. Stroman, et al., Astrophys. J. 684, 1174 (2008).

20. G. Pelletier, M. Lemoine, and A. Marcowith, Astron. Astrophys. 453, 181 (2006).

21. B. Reville, J.G. Kirk, P. Duffy, et al., Astron. Astrophys. 475, 435 (2007).

22. S. P. Reynolds, Ann. Rev. Astron. Astrophys. 46, 89 (2008).

23. S. P. Reynolds, K. J. Borkowski, D. A. Green, et al., Astrophys. J. 680, L41 (2008).

24. D. Ryu, H. Kang, J. Cho, and S. Das, Science 320 , 909 (2008).

25. R. Z. Sagdeev, Problems of the Plasma Theory, Ed. by M. A. Leontovich (Atomizdat, Moscow, 1964), Vol. 4, p. 20 [in Russian].

26. J. Skilling, Mon. Not. R. Astron. Soc. 173, 255 (1975).

27. A. Spitkovsky, Astrophys. J. 682, L5 (2008).

28. J. B. Taylor, Rev. Mod. Phys. 58, 741 (1986).

29. I. N. Toptygin, Cosmic Rays in Interplanet Magnetic Fields (Nauka, Moscow, 1983) [in Russian].

30. Y. Uchiyama, F. A. Aharonian, T. Tanaka, et al., Nature 449, 576 (2007).

31. S. I. Vainshtein, Ya. B. Zel'dovich, and A. A. Ruzmaikin, Turbulent Dynamo in Astrophysics (Nauka, Moscow, 1980), p. 31 [in Russian].

32. A. E. Vladimirov, A. M. Bykov, and D. C. Ellison, Astrophys. J. 688, 1084 (2008).

33. A. Vladimirov, D. Ellison, and A. Bykov, Astrophys. J. 652, 1246 (2006).

34. E. S. Weibel, Phys. Rev. Lett. 2, 83 (1959).

35. V. N. Zirakashvili and V. S. Ptuskin, Astrophys. J. 678, 939 (2008).

Translated by V. Astakhov 